\title{
Neurofibromin Expression is Associated with Aggressive Disease and Poor Outcome in Colorectal Carcinoma
}

\author{
ADAM ELZAGHEID $^{1,2}$, FATMA EMAETIG ${ }^{1}$, WESAM ELSAGHAYER $^{3}$, FAIROUZ TORJMAN $^{4}$, \\ MATTI LATTO $^{5}$, KARI SYRJÄNEN ${ }^{6,8}$, YRJÖ COLLAN ${ }^{7}$ and SEPPO PYRHÖNEN ${ }^{6}$ \\ ${ }^{1}$ Department of Pathology, Faculty of Medicine, Benghazi University, Benghazi, Libya; \\ ${ }^{2}$ Department of Genetic Engineering, Biotechnology Research Center, Tripoli, Libya; \\ ${ }^{3}$ Department of Pathology, Faculty of Medicine, Misurata University, Misurata, Libya; \\ ${ }^{4}$ Department of Pathology, Faculty of Medicine, Tripoli University, Tripoli, Libya; \\ Departments of ${ }^{5}$ Surgery, ${ }^{6}$ Oncology and Radiotherapy and \\ ${ }^{7}$ Pathology, Turku University Hospital and University of Turku, Turku, Finland; \\ ${ }^{8}$ Teaching and Research Institute, Barretos Cancer Hospital, Barretos-SP, Brazil
}

\begin{abstract}
Aim: To assess the predictive and prognostic value of neurofibromin (NF) expression in colorectal carcinoma $(C R C)$. Materials and Methods: The present series consists of archival samples from 191 patients with stage I, II, III, or IV CRC treated between 1981 and 1990 at the Turku University Hospital (Finland). Tumor biopsies as microarray blocks were analyzed for expression of NF by immunohistochemistry. Different grading systems were tested for NF expression. Results: A significant correlation between NF expression and tumor localization was found, with tumors arising in the colon showing intense NF expression more often than those arising in the rectum ( $p=0.014)$. Higher expression of $N F$ was more common in tumors not responding to treatment $(p=0.004)$. Tumors with multiple metastases showed higher expression of $N F$ than those with single metastasis only $(p=0.025)$. Furthermore, NF expression showed a borderline $(p=0.068)$ correlation with gender; tumors of women showed higher NF expression that those of males. On the other hand, NF expression was not significantly associated with tumor recurrence, age, lymph node involvement, tumor grade and tumor stage or disease outcome. Conclusion: Positive NF expression in CRC is a sign of aggressive disease and poor outcome.
\end{abstract}

This article is freely accessible online.

Correspondence to: Adam Elzagheid, MD, Ph.D., Professor, Department of Genetic Engineering, Biotechnology Research Center, Tripoli, Libya. Tel: +218 914461615, Fax: +218 213600035, e-mail: elzagheid@yahoo.com

Key Words: Neurofibromin (NF) expression, immunohistochemistry, prognosis, treatment, adjuvant therapy.
Colorectal cancer (CRC) is the third most common cancer in the world, also ranking as the third most common cause of cancer-related deaths (1). Despite the fact that most CRC patients undergo potentially curative surgery and adjuvant chemotherapy administered, approximately $50 \%$ of all patients initially considered curable by surgery, will subsequently relapse and die of their disease (2).

The etiology of CRC remains open and the overall outcome is poor (3). However, some fundamental advances in our understanding of the biology and genetics of CRC have been made recently (4-5).

CRC arises from progressive accumulation of genetic and epigenetic changes (e.g. chromosomal instability, microsatellite instability and methylation pathway that cause the transformation of normal colon mucosa cells into adenocarcinoma $(6,7)$.

Alterations in NF1 gene mutations and neurofibromin (NF) expression have been found in colon adenocarcinoma, myelodysplastic syndrome, anaplastic astrocytoma and small-cell lung carcinoma (8-9). NF, the protein encoded by NF 1 gene, is a cytoplasmic protein predominantly expressed in neurons, Schwann cells, oligodendrocytes and leucocytes (10). It functions as a GTPase, activating protein (GAP) for Ras by accelerating the conversion of active Ras-GTP to inactive Ras-GDP $(11,12)$. NF acts as a negative regulator of the Ras/mitogen-activated protein kinase signaling pathway via suppression of active Ras (13). Therefore, the negative regulation of Ras activity classifies $N F 1$ gene as a tumor suppressor gene.

To date, little is known about the role of tumor suppressor $\mathrm{NF}$ in $\mathrm{CRC}$, and data on its potential prognostic value in these tumors are lacking. In this study, we examined the expression of NF in $191 \mathrm{CRCs}$ using immuno-histochemistry 
(IHC) and correlated the results with the established clinicopathological variables and outcome of the disease.

\section{Materials and Methods}

The present series consists of tissue samples obtained from 191 patients with stage I, II, III, or IV CRC who underwent bowel resection during 1981 and 1990 at Turku University Hospital (TUH, Finland), available for study at the archives of the Department of Pathology. IHC staining was performed at the Department of Pathology, Benghazi University, Benghazi, Libya. All pertinent clinical and histopathological data of the patients were collected from the patients' case records as summarized in Table I. All patients have been prospectively followed-up until death or when last seen alive on their clinical visit (March 2007), with a median follow-up time of 77 months (range=2-263). The study was approved by the TUH Ethics Committee and conducted in accordance with the endorsement of the National Authority for Medico-legal Affairs

Tissue microarray. Archival paraffin-embedded samples were used to build up tissue microarray (TMA) blocks for IHC staining. Areas of invasive tumor with the lowest degree of differentiation, abundant in cells with the highest number of mitoses, were chosen from the original blocks. Necrotic and autolytic areas and areas containing predominantly stromal tissue were excluded. For tumors producing abundant intra- or extracellular mucin, invasive areas with the highest number of epithelial cells were chosen. These representative areas were marked by an experienced pathologist on hematoxylin and eosin (HE)-stained slides from selected paraffin blocks and a cylinder of tissue $(1 \mathrm{~mm}$ in diameter) was cut with a TMA instrument (Beecher Instruments, Sun Prairie, WI, USA) into a new paraffin block. This size of tissue section (1-mm wide) was equal to the often used three cores, 0.6-mm wide (20-23). As the core was larger than usual, sampling differences were less in $0.6-\mathrm{mm}$ cores. Serial $4-\mu \mathrm{m}$ sections were then cut from the TMA paraffin blocks. The sections were mounted on ChemMate ${ }^{\mathrm{TM}}$ Capillary Gap plus Slides (gray) (Dako, Glostrup, Denmark). Normal colorectal mucosa was selected at least $2 \mathrm{~mm}$ apart from the malignant tissues of the section. If available, another normal sample was obtained from normal colorectal mucosa at either of the resection margins in the surgical specimens. On average, two normal controls were available. Lymphatic follicles and hyperplastic, as well as inflamed areas were avoided. To obtain enough mucosa for tissue array, all tangentially cut areas were avoided.

Neurofibromin (NF) immunostaining. Immunohistochemical (IHC) analysis was done using an automated system (BenchMark XT; Ventana Medical System, Inc., Tucson, AZ, USA). This fully automated processing of code-labeled slides included baking of the slides, solvent-free deparaffinization, antigen retrieval in the cell conditioning buffer $\mathrm{CC} 1$ (Mild: 36 minutes conditioning and standard: 60 minutes conditioning), incubation with rabbit monoclonal anti-neurofibromin antibody, $2.0 \mathrm{ml}$ ready-to-use from Spring Bioscience at a dilution of $1: 50$ for 32 minutes, at $37^{\circ} \mathrm{C}$ (clone: NF n27b, Catalog No: M3794; Parkway, CA, USA), as well as application of ultra viewTM universal DAB inhibitor, ultraview universal DAB chromogen, ultraview universal DAB $\mathrm{H}_{2} \mathrm{O}_{2}$, ultraview universal DAB copper and ultraview universal HRP multimer.
Table I. Clinicopathological characteristics of patients.

\begin{tabular}{|c|c|}
\hline Characteristic & Number of patients $(\%)$ \\
\hline \multicolumn{2}{|l|}{ Gender } \\
\hline Male & $93(48.7 \%)$ \\
\hline Female & $98(51.3 \%)$ \\
\hline \multicolumn{2}{|l|}{ Age (years) } \\
\hline$<65$ years & $87(45.5 \%)$ \\
\hline$>65$ years & $104(54.5 \%)$ \\
\hline \multicolumn{2}{|l|}{ Primary tumor status } \\
\hline $\mathrm{T} 1$ & $5(2.6 \%)$ \\
\hline $\mathrm{T} 2$ & $14(7.3 \%)$ \\
\hline $\mathrm{T} 3$ & $114(59.7 \%)$ \\
\hline $\mathrm{T} 4$ & $58(30.4 \%)$ \\
\hline \multicolumn{2}{|l|}{ LN involvement } \\
\hline No & $122(63.9 \%)$ \\
\hline Yes & $69(36.1 \%)$ \\
\hline \multicolumn{2}{|l|}{ Stage } \\
\hline I & $19(10 \%)$ \\
\hline II & $103(54 \%)$ \\
\hline III & $30(15.6 \%)$ \\
\hline IV & $39(20.4 \%)$ \\
\hline \multicolumn{2}{|l|}{ Histological grade } \\
\hline Grade I & $27(14.1 \%)$ \\
\hline Grade II & $140(73.3 \%)$ \\
\hline Grade III & $24(12.6 \%)$ \\
\hline \multicolumn{2}{|l|}{ Localization } \\
\hline Right colon & $65(34 \%)$ \\
\hline Left colon & $69(36 \%)$ \\
\hline Rectum & $57(30 \%)$ \\
\hline \multicolumn{2}{|l|}{ Distant Metastasis } \\
\hline No & $152(79.6 \%)$ \\
\hline Yes & $39(20.4 \%)$ \\
\hline \multicolumn{2}{|l|}{ Recurrence during the follow-up } \\
\hline Yes & $56(29.3 \%)$ \\
\hline No & $96(50.3 \%)$ \\
\hline Unknown & $39(20.4 \%)$ \\
\hline \multicolumn{2}{|l|}{ Status at the end of follow-up } \\
\hline Alive & $50(26.2 \%)$ \\
\hline Dead as result of disease & $87(54.5 \%)$ \\
\hline Dead from other cause (s) & $54(28.3 \%)$ \\
\hline \multicolumn{2}{|l|}{ Response to therapy } \\
\hline Response & $9(4.7 \%)$ \\
\hline No response & $28(14.7 \%)$ \\
\hline Unknown & $154(80.6 \%)$ \\
\hline
\end{tabular}

Counterstaining with hematoxylin II (C00758) was performed for 4 min followed by post-counterstaining with blueing reagent (B11129) for $4 \mathrm{~min}$. After staining, the sections were dehydrated in ethanol, cleared in xylene and covered with Mountex and coverslips.

Evaluation of NF staining. NF staining was evaluated using regular light microscope at the magnification of $\mathrm{x} 40$, blinded by the information on tumor grade, stage or clinical outcome. Membranous and cytoplasmic staining were evaluated separately. Two different grading (A and B) systems were applied to assess the patterns of $\mathrm{NF}$ expression in tumor cells. In system A, the staining was graded 
a

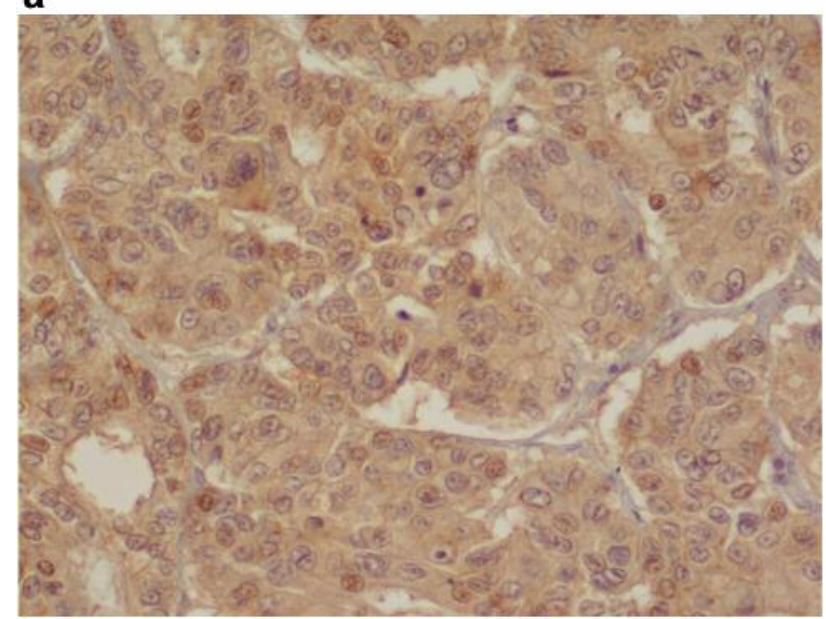

b

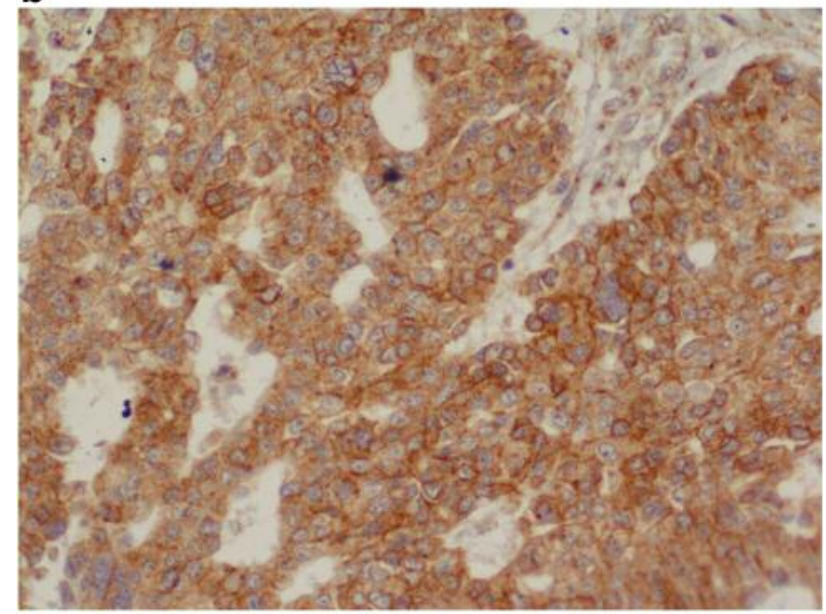

Figure 1. a: Weak neurofibromin expression in CRC ( $\times 40$ magnification). $b$ : Moderate and strong neurofibromin expression in CRC ( $\times 40$ magnification).

into four categories: 0, no expression (no detectable staining); 1 , weak staining; 2 , moderate staining; and 3, strong staining intensity. In system B, staining was graded in two categories: 1 , no/weak expression; and 2, moderate/strong expression. Both systems were statistically tested.

In calculating the staining indexes, cytoplasmic and membranous index, the intensity of staining and the fraction of positively stained cells were taken into account using the following formula:

$\mathrm{I}=0 \times \mathrm{f} 0+1 \times \mathrm{f} 1+2 \times \mathrm{f} 2+3 \times \mathrm{f} 3$

Where "I" is the staining index and f0-f3 are the fractions of the cells showing a defined level of staining intensity (from 0 to 3 ). Theoretically, the index could vary between 0 and $3(14,15)$.

Statistical analysis. SPSS for Windows SPSS 19.0.1 (IBM, Armonk, NY, USA) was used for statistical analysis. Frequency tables were analyzed using the Chi-square test, with Fisher's

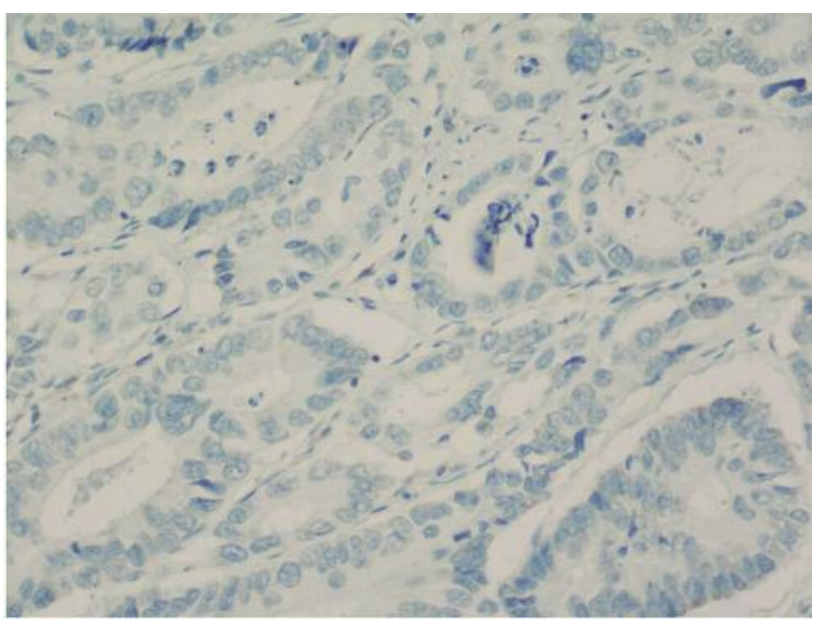

Figure 2. Negative neurofibromin expression in CRC ( $\times 40$ magnification).

exact test (where appropriate), or likelihood ratio (LR) statistics to assess the significance between categorical variables. Differences in the means of continuous variables were analyzed using analysis of variance ANOVA or non-parametric tests (Mann-Whitney, Kruskal-Wallis) tests. Reported $p$-values are from two-sided tests; in all analyses, $p<0.05$ was regarded as statistically significant.

\section{Results}

Description of NF expression patterns. The expression pattern of NF was cytoplasmic and membranous in the tumor area. Examples of the staining patterns of NF are illustrated in Figures 1a, 1b and 2. Of the 191 tumors, 176 (92.1\%) were considered positive (staining intensity $+1,+2,+3$; Figures $1 \mathrm{a}, \mathrm{b})$, whereas $15(7.9 \%)$ were considered negative (staining intensity 0 ; Figure 2 ).

NF expression correlates with clinicopathological features. A significant correlation between NF expression and tumor localization was found, tumors arising in the colon showed intense NF expression more often than tumors arising in the rectum $(p=0.014)$.

NF expression with higher intensity was more common in tumors not responding to treatment $(p=0.004)$. Also tumors with multiple metastases expressed NF more intensely than tumors with single metastasis only $(p=0.025)$. Furthermore, NF expression showed a borderline $(p=0.068)$ correlation with gender, with tumors of the women showing higher NF expression than tumors of men. On the other hand, NF expression was not significantly associated with tumor recurrence, age, lymph node involvement, tumor grade and tumor stage or disease outcome at study endpoint (Table II). 
Table II. Correlation between neurofibromin expression and clinicopathological features of colorectal carcinoma.

\begin{tabular}{|c|c|c|c|c|c|c|}
\hline \multirow[t]{2}{*}{ Features } & \multirow[t]{2}{*}{ Number of cases $(\%)$} & \multicolumn{4}{|c|}{ Neurofibromin expression } & \multirow[b]{2}{*}{$p$-Value } \\
\hline & & Negative & Weak & Moderate & Strong & \\
\hline Gender & & & & & & 0.195 \\
\hline Male & $93(48.7 \%)$ & $7(7.5 \%)$ & $26(28 \%)$ & $34(36.6 \%)$ & $26(28 \%)$ & \\
\hline Female & $98(51.3 \%)$ & $8(8.2 \%)$ & $15(15.3 \%)$ & $45(45.9 \%)$ & $30(30.6 \%)$ & \\
\hline Age group (years) & & & & & & 0.229 \\
\hline$<65$ & $87(45.5 \%)$ & $8(9.2 \%)$ & $15(17.2 \%)$ & $33(37.9 \%)$ & $31(35.6 \%)$ & \\
\hline$>65$ & $104(54.5 \%)$ & $7(6.7 \%)$ & $26(25.0 \%)$ & $46(44.2 \%)$ & $25(24.0 \%)$ & \\
\hline Primary tumor status & & & & & & 0.204 \\
\hline $\mathrm{T} 1$ & $5(2.6 \%)$ & $0(0 \%)$ & $0(0 \%)$ & $2(40 \%)$ & $3(60 \%)$ & \\
\hline $\mathrm{T} 2$ & $14(7.3 \%)$ & $1(7.1 \%)$ & $5(35.7 \%)$ & $4(28.6 \%)$ & $4(28.6 \%)$ & \\
\hline $\mathrm{T} 3$ & $114(59.7 \%)$ & $7(6.1 \%)$ & $27(23.7 \%)$ & $53(46.5 \%)$ & $27(23.7 \%)$ & \\
\hline $\mathrm{T} 4$ & $58(30.4 \%$ & $7(12.1 \%)$ & $9(15.5 \%)$ & $20(34.5 \%)$ & $22(37.9 \%)$ & \\
\hline $\mathrm{LN}$ involvement & & & & & & 0.299 \\
\hline No & $122(63.9 \%)$ & $10(8.2 \%)$ & $25(20.5 \%)$ & $56(45.9 \%)$ & $31(25.4 \%)$ & \\
\hline Yes & $69(36.1 \%)$ & $5(7.2 \%)$ & $16(23.2 \%)$ & $23(33.3 \%)$ & $25(36.2 \%)$ & \\
\hline Distant metastasis & & & & & & 0.190 \\
\hline No & $152(79.6 \%)$ & $12(7.9 \%)$ & $34(22.4 \%)$ & $67(44.1 \%)$ & $39(25.7 \%)$ & \\
\hline Yes & $39(20.4 \%)$ & $3(7.7 \%)$ & $7(17.9 \%)$ & $12(30.8 \%)$ & $17(43.6 \%)$ & \\
\hline Stage & & & & & & 0.406 \\
\hline I & $19(10 \%)$ & $1(5.3 \%)$ & $5(26.3 \%)$ & $6(31.6 \%)$ & $7(36.8 \%)$ & \\
\hline II & $103(54 \%)$ & $9(8.7 \%)$ & $20(19.4 \%)$ & $50(48.5 \%)$ & $24(23.3 \%)$ & \\
\hline III & $30(15.6 \%)$ & $2(6.7 \%)$ & $9(30 \%)$ & $11(36.7 \%)$ & $8(26.7 \%)$ & \\
\hline IV & $39(20.4 \%)$ & $3(7.7 \%)$ & $7(17.9 \%)$ & $12(30.8 \%)$ & $17(43.6 \%)$ & \\
\hline Histological grade & & & & & & 0.895 \\
\hline Grade I & $27(14.1 \%)$ & $2(7.4 \%)$ & $7(25.9 \%)$ & $9(33.3 \%)$ & $9(33.3 \%)$ & \\
\hline Grade II & $140(73.3 \%)$ & $11(7.9 \%)$ & $30(21.4 \%)$ & $61(43.6 \%)$ & $38(27.1 \%)$ & \\
\hline Grade III & $24(12.6 \%)$ & $2(8.3 \%)$ & $4(16.7 \%)$ & $9(37.5 \%)$ & $9(37.5 \%)$ & \\
\hline Localization & & & & & & 0.014 \\
\hline Colon & $134(70 \%)$ & $12(9 \%)$ & $23(17.2 \%)$ & $64(47.8 \%)$ & $35(26 \%)$ & \\
\hline Rectum & $57(30 \%)$ & $3(5.3 \%)$ & $18(31.6 \%)$ & $15(26.3 \%)$ & $21(36.8 \%)$ & \\
\hline Recurrence & & & & & & 0.482 \\
\hline Yes & $56(29.3 \%)$ & $6(10.7 \%)$ & $11(19.6 \%)$ & $22(39.3 \%)$ & $17(30.4 \%)$ & \\
\hline No & $96(50.3 \%)$ & $6(6.3 \%)$ & $23(24 \%)$ & $45(46.9 \%)$ & $22(22.9 \%)$ & \\
\hline Unknown & $39(20.4 \%)$ & No statistical data & No statistical data & No statistical data & No statistical data & \\
\hline Status at the end of FU & & & & & & 0.937 \\
\hline Alive & $50(26.2 \%)$ & $4(8 \%)$ & $10(20 \%)$ & $21(42 \%)$ & $15(30 \%)$ & \\
\hline Dead as result of disease & $87(54.5 \%)$ & $8(9.2 \%)$ & $15(17.2 \%)$ & $35(40.2 \%)$ & $29(33.3 \%)$ & \\
\hline Dead from other causes & $54(28.3 \%)$ & No statistical data & No statistical data & No statistical data & No statistical data & \\
\hline Response to therapy & & & & & & 0.004 \\
\hline Response & $9(4.7 \%)$ & $4(44.4 \%)$ & $2(22.2 \%)$ & $2(22.2 \%)$ & 1(11.2) & \\
\hline No response & $28(14.7 \%)$ & $0(0 \%)$ & $7(25 \%)$ & $13(46.4 \%)$ & $8(28.6 \%)$ & \\
\hline Unknown & $154(80.6 \%)$ & No statistical data & No statistical data & No statistical data & No statistical data & \\
\hline Multiple Distant Metastases & $12(31 \%)$ & $0(0 \%)$ & $1(8 \%)$ & $5(42 \%)$ & $6(50 \%)$ & 0.025 \\
\hline
\end{tabular}

Survival analysis. In the Kaplan-Meier survival analysis, there was a difference in recurrence-free survival (RFS) or disease-free survival (DFS) between patients with tumors expressing no/weak NF and those with NF overexpression $(p=0.149)$ (Figure 3). Interestingly, at 3-year follow-up, 30\% of the patients with tumors overexpressing NF showed recurrence as compared to only $15 \%$ of the patients whose tumors showed no/weak expression.

\section{Discussion}

This study is a continuation of our efforts to further elucidate the biology of CRC and identify more effective prognostic factors. Colon cancer arises from progressive accumulation of genetic and epigenetic changes that causes the transformation of normal colon mucous cells into adenocarcinoma $(6,16)$. The role of $N F 1$ gene or its protein 


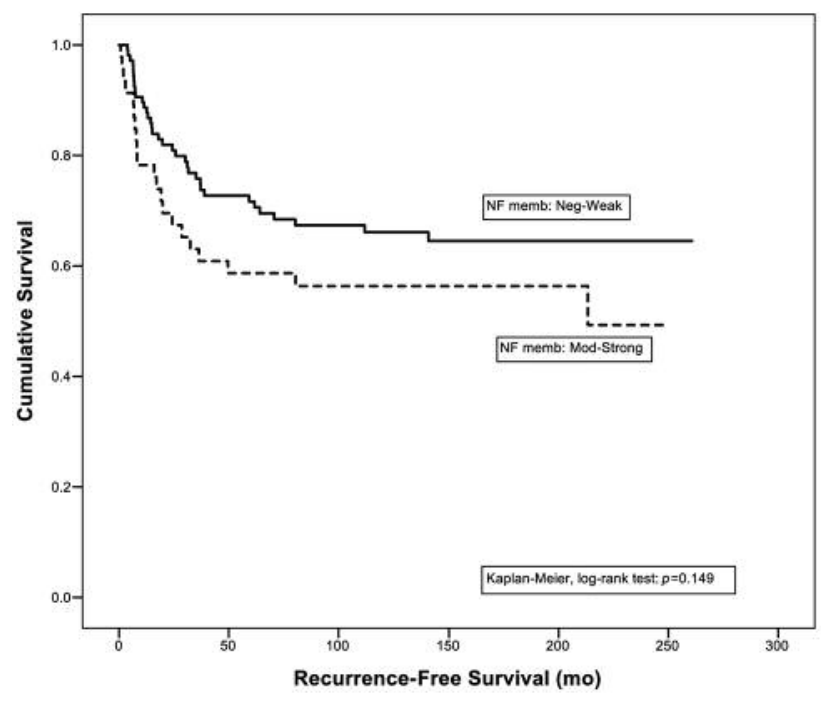

Figure 3. Neurofibromin expression (neg-weak vs. mod-strong) as determinant of disease-free survival in univariate (Kaplan-Meier) analysis ( $p=0.149$ log-rank).

NF in the development and progression of colon cancer is unknown. The aim of this study was to examine the role of tumor suppressor NF in CRC. We examined the expression of NF by IHC in 191 cases of CRC. NF expression was detected in $92.1 \%$ of $\mathrm{CRC}$ cases and found to correlate significantly with tumor localization, tumors not responding to treatment, tumors with multiple metastases and those (less significantly) to gender. In contrast, no correlation was established with tumor recurrence, age, lymph node involvement, tumor grade and stage.

Two different grading systems were applied to assess the patterns of neurofibromin expression. In system A, a 4-tier grading (of $0,1,2,3 ;$ ) (no, weak, moderate, strong staining intensity, respectively) was used, which was shown to correlated significantly with tumors' localization $(p=0.014)$, and tumors not responding to treatment $(p=0.004)$. On the other hand, this grading failed to correlate with tumor recurrence, age, gender, lymph node involvement, distant metastases, tumor grades and stages.

In system B, a 2-tier grading 0/1 vs. 2/3 (negative/weak $v s$. moderate/strong staining intensity) was used and shown to correlate significantly with failing treatment response $(p=0.042)$, multiple metastases $(p=0.025)$, and (borderline significantly) with gender. Similarly, no correlation was found with tumor localization, tumor recurrence, age, lymph node involvement, tumor grades and stages.

Interestingly, we observed a significant correlation between NF expression and tumor localization; positive expression of $\mathrm{NF}$ was significantly $(p=0.014)$ associated with left-sided tumors, whereas NF expression was lost more frequently in right-sided colon cancers. This suggests that there may be differences between the normal right and left colonic segments that could favor malignant transformation through different molecular pathways. Such differences are probably related to different molecular profiles of tumors, microsatellite instability, and methylator phenotypes being associated with right-sided tumors, as well as chromosomal instability with left-sided tumors $(17,18)$. Future molecular studies are necessary to confirm this hypothesis.

In conclusion, the present work showed NF expression to be significantly associated $(p=0.004)$ with poor response to treatment and tumors with multiple metastases $(p=0.025)$, suggesting that positive NF expression in CRC might be an indicator of aggressive disease and poor outcome. However, larger series of CRCs need to be analyzed to validate these observations.

\section{Acknowledgements}

This project was supported by grants from the National Agency for Scientific Research (NASR), Tripoli, Libya.

\section{References}

1 Jemal A, Bray F, Center MM, Ferlay J, Ward E and Forman D: Global cancer statistics. CA Cancer J Clin 61: 69-90, 2011.

2 Higa R: Colorectal cancer: Epidemiology and primary prophylaxis. Acta Gastroenterol Latinoam 41: 70-73, 2011.

3 Fearon ER and Vogelstein B: A genetic model for colorectal tumorigenesis. Cell 61: 759-767, 1990.

4 Luebeck EG and Moolgavkar SH: Multistage carcinogenesis and the incidence of colorectal cancer. Proc Natl Acad Sci USA 99: 15095-15100, 2002.

5 Kinzler K and Vogelstein B: Lessons from hereditary colorectal cancer. Cell 87: 159-70, 1996.

6 Fodde R: The APC gene in colorectal cancer Eur J Cancer 38: 867-871, 2002.

7 Trovo MAB and Tajara EH: Neurofibromin: A general outlook. Clin Genet 70: 1-13, 2006.

8 Hall A: Signal transduction through small GTPases - a tale of two GAPs. Cell 69: 389-391, 1992.

$9 \mathrm{Xu}, \mathrm{GO}$, Connell P, Viskochil D, Gawthon R, Robertson M, Culver M, Dunn D, Stevens J, Gesteland R and White R: The neurofibromatosis type I gene encodes a protein related to GAP. Cell 62: 599-608, 1990.

10 Van Spyk SL, Thomas N, Cooper DN and Upadhyaya M: Neurofibromatosis type I- associated tumors: Their somatic mutational spectrum and pathogenesis. Hum Genomics 5: 623690, 2011.

11 Andersen LB, Fountain JW, Gutmann DH, Tarle SA, Glover TW, Dracopoli NC, Housman DE and Collins FS: Mutations in the neurofibromatosis 1 gene in sporadic malignant melanoma cell lines. Nat Genet 3(2): 118-121, 1993.

12 Foster WJ, Fuller CE, Perry A and Harbor JW: Status of the NF1 tumor suppressor locus in uveal melanoma. Arch Opthalmol 121(9): 1311-1315, 2003. 
13 Staib L, Link KH, Blatz A and Beger HG: Surgery of colorectal cancer: Surgical morbidity and five-and ten year results in 2,400 patients - mono-institutional experience. World J Surg 26: 5966, 2002.

14 Elzagheid A, Emaetig F, Alkikhia L ,Buhmeida A, Syrjanen K, El faitori O, Latto $\mathrm{M}$, Collan $\mathrm{Y}$ and Pyrhonen S: High cyclooxygenase-2 expression is associated with advanced stages in colorectal cancer. Anticancer Res 33(8): 3137-3143, 2013.

15 Kaur A, Elzagheid A, Birkman EM, Avoranta T, Kylola V, Korkeila E, Syrjanen K, Westermarck J and Sundstrom J: Protein phosphatase methylesterase-1 (PME-1) expression predicts a favorable clinical outcome in colorectal cancer. Cancer Med 4(12): 1798-1808, 2015.

16 Kinzler K and Vogelstein B: Lessons from hereditary colorectal cancer. Cell 87: 159-170, 1996.
17 Bendardaf R, Lamlum H, Ristamäki R, Algars A, Collan Y and Pyrhonen S: Response to chemotherapy (irinotecan plus 5fluorouracil) in colorectal carcinoma can be predicted by tumor DNA content. Oncol 66(1): 46-52, 2004.

18 Bendardaf R, Lamlum H, Ristamäki R, Korkeila E, Syrijanen K and Pyrhonen S: Thymidylate synthase and microsatellite instability in colorectal cancer: Implications for disease-free survival, treatment response and survival with metastases. Acta Oncol 47(6): 1046-1053, 2008.
Received August 12, 2016

Revised August 27, 2016

Accepted August 30, 2016 